\title{
Design of Switched Quantizers and Speech Coding Based on Quasi-Logarithmic Compandor
}

\author{
Nikola J. Vucic ${ }^{1}$, Zoran H. Peric ${ }^{1}$, Goran M. Petkovic ${ }^{2}$ \\ ${ }^{I}$ Faculty of Electronic Engineering, University of Nis, \\ Aleksandra Medvedeva 14, 18000 Nis, Serbia \\ ${ }^{2}$ College of Applied Studies, \\ Filipa Filipovica 20, 17500 Vranje, Serbia \\ zoran.peric@elfak.ni.ac.rs
}

\begin{abstract}
This article investigates switched quantizers for speech signal depicted with Gaussian probability density function (PDF). Gaussian PDF is better for smaller frame lengths that are represented here. Companding technique results in constant Signal to Quantization Noise Ratio (SQNR). In this paper two approaches are present: quasi-logarithmic (QL) and piecewise uniform (PU) compandor. Simpler compandor directly affects the complexity of hardware realization and expense of given solution, but, on the other hand, also brings to weaker performances. Therefore, a smart choice has to be made. Usage of switched technique leads to better performances. This way, the quality of quantization is improved by dividing the dynamic range of variances into multiple subranges. For each subrange a separate quantizer is designed, with its support region's amplitude. The optimal amplitude is numerically determined, whereby a single criterion is obtaining the maximal SQNR. Bit rates of these quantizers don't depend on signal variance, as the fixed length codes are used. The performances of proposed quantizers are demonstrated on real speech signals from the reliable database. Comparison of obtained results with other recent solutions is done in order to show the efficiency of this model.
\end{abstract}

Index Terms-Signal processing algorithms; Signal quantization; Speech processing; Signal to noise ratio.

\section{INTRODUCTION}

The design of quantizers for speech signal transmission mostly assumes that input signal can be well described with Gaussian or Laplacian probability density functions (PDF). Both approaches are represented in the recent researches [1]-[4]. A quantizer designed according to the Gaussian PDF leads to better performances, i.e. higher Signal to Quantization Noise Ratio (SQNR), than a quantizer with Laplacian PDF. Another approach is a frame size: the quantizer with Gaussian PDF is used for smaller frames [5]. Since this is the case in our paper, we will present a model with Gaussian PDF. Every quantizer is described with its codebook with a certain number of code words. They divide

Manuscript received 3 January, 2018; accepted 11 August, 2018.

This work was supported by the Ministry of Education, Science and Technological Development of the Republic of Serbia under the project TR 32035 . into fixed length codes (FLC), analysed in [1], and variable length codes (VLC), examined in [2], [3]. In this research, we put a stress on FLC, whereby the code length is $\log _{2} N$ for the codebook with $N$ levels. Paper [2] proposes a model of QL quantizer with VLC; and [3] investigates piecewise uniform quantizer with $N=128$ levels. Switched quantizers are elaborated in [2], [3], as the division of variance range into subranges, whereby for each subrange separate quantizer is designed, brings to the achievement of better performances. QL compandor is characterized with its nonlinear compression function, and PU compandor with linear approximation, i.e. linear splines, between samples.

The switched piecewise uniform quantizer's reduced complexity of designing and hardware realization gave us motivation to work on this paper. Here are also proposed both efficient approximations of numerical $Q$ function, and methods of computing performances of piecewise uniform quantizer [6]. In order to prove the efficiency of proposed solutions, we performed their software simulations on real speech signals from [7].

\section{SWITCHED SCALAR QUASI-LOGARITHMIC QUANTIZER}

In order to suppress mismatch of the weak signal to the uniform quantizer, a non-uniform quantization has been developed. One of the realization methods was proposed by [5], by introducing companding technique, which works as follows: first, input signal is being compressed with nonlinear compressor whose compression function is $c(x)$, afterwards obtained compressed signal is quantized with uniform quantizer $Q(x)$, and at the end, expanding, with an inverse compression function $c^{-1}(x)$, is applied. The block diagram of this method is illustrated on Fig. 1.

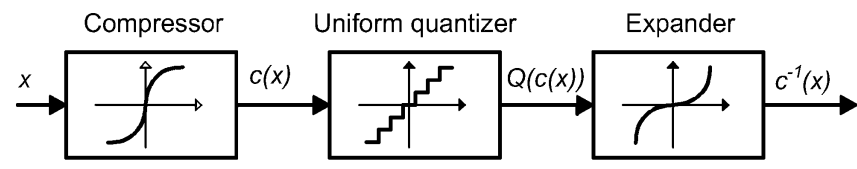

Fig. 1. Block diagram of the companding technique.

In order to achieve constant Signal to Quantization Noise Ratio (SQNR) in the wide range of input variances, quasi- 
logarithmic (QL) $\mu$-law of compression can be applied

$$
c(x)=x_{\max } \frac{\ln \left(1+\mu|x| / x_{\max }\right)}{\ln (1+\mu)} \operatorname{sgn}(x),
$$

where $\mu$ is a non-dimensional compression factor, and $x_{\max }$ is a support region's amplitude. The support region of scalar compandor is $\left[-x_{\max }, x_{\max }\right]$. Due to its symmetry, we are able to make some analyses just on the positive part. Determining the optimal support region's amplitude is very important for the compandor's design and serious examinations need to be done. The uniform quantizer is realized with $N$ levels. The support region is divided into $2 l=8$ segments, which are further divided into unequal cells. In total there are $N-2$ cells, whose lower and upper borders are $x_{i-1}$ and $x_{i}$, respectively, whereby $i=2, \ldots, N-1$. Edge borders are $x_{1}=$ $-x_{\max }$ and $x_{N-1}=x_{\max }$, and for full examination is set: $x_{0} \rightarrow$ $-\infty, x_{N} \rightarrow \infty$. Each of these cells has its representation level $y_{i}$. All samples bigger than $x_{\max }$ are represented with $y_{N}$, and all samples smaller than $-x_{\max }$ are represented with $y_{1}=-y_{N}$. The final element of this companding technique, expander, uses inverse compression function $c^{-1}(x)$. A mathematically complex inverse function would imply more expensive compandor's hardware realization, so it's important to apply suitable compression function $c(x)$.

Speech signal can be depicted with Gaussian probability density function

$$
p(x)=\frac{1}{\sqrt{2 \pi} \sigma} \exp \left(-\frac{x^{2}}{2 \sigma^{2}}\right)
$$

Distortion, as a measure of quantizer's quality, is defined as expected mean squared error between original and quantized signal. Granular distortion $D_{\mathrm{g}}$ is calculated within the support region $\left[-x_{\max }, x_{\max }\right]$. The overload distortion $D_{\mathrm{ov}}$ measures performances out of that region. Total distortion is sum of these distortions: $D=D_{\mathrm{g}}+D_{\mathrm{ov}}$, that are equal to:

$$
\begin{gathered}
D_{\mathrm{g}}=\frac{\ln ^{2}(1+\mu)}{3 N^{2}} \sigma^{2}\left[\frac{x_{\max }^{2}}{\mu^{2} \sigma^{2}}+\frac{2 x_{\max }}{\mu \sigma^{2}}|\bar{x}|+\frac{\overline{x^{2}}}{\sigma^{2}}\right], \\
D_{\mathrm{ov}}=2 \int_{x_{\max }}^{\infty}\left(x-y_{N}\right)^{2} p(x) d x,
\end{gathered}
$$

where $\overline{|x|}$ and $\overline{x^{2}}$ are defined as:

$$
\left\{\begin{array}{l}
\mid \overline{|x|}=2 \int_{0}^{x_{\max }} x p(x) d x, \\
\overline{x^{2}}=2 \int_{0}^{x_{\max }} x^{2} p(x) d x .
\end{array}\right.
$$

For large $x_{\max }$ and assuming $y_{N} \cong x_{\max }$ we can derive:

$$
D_{\mathrm{g}}=\frac{\ln ^{2}(1+\mu)}{3 N^{2}} \sigma^{2}\left\lfloor 1+\frac{c^{2}}{\mu^{2}}+\frac{2 c}{\mu} \sqrt{\frac{2}{\pi}}\right\rfloor,
$$

$$
\begin{gathered}
D_{\mathrm{ov}}= \\
=\sigma^{2}\left[\left(1+c^{2}\right)\left(1-\operatorname{erf}\left(\frac{c}{\sqrt{2}}\right)\right)-c \exp \left(-\frac{c^{2}}{2}\right) \sqrt{\frac{2}{\pi}}\right]
\end{gathered}
$$

where parameter $c=x_{\max } / \hat{\sigma}_{p}$ is quantizer's relative range factor.

We can further calculate Signal to Quantization Noise Ratio- SQNR $[\mathrm{dB}]$ as $S Q N R=10 \log \left(\sigma^{2} / D\right)$.

Although QL quantizer provides almost constant SQNR in the wide dynamic range of variances, even better results can be achieved with switched quantization technique. The quality of quantization is improved by dividing the dynamic range of variances into multiple subranges. Each of these subranges has its specially adapted compression function. The most used adaptation parameter is an average power of the signal, i.e. variance of the speech signal. If processing method of input samples "frame by frame" is used for quantization, a goal is to achieve the highest quality in the wide supposed range of frame variances.

Switched technique starts with buffering $j$-th frame that has $M$ samples $x_{j, i}$, whereby $i=1, \ldots, M$, as shown in Fig. 2 . After buffering, a variance of $j$-th frame is being determined and then log-uniform quantized. On our disposal are $k$ quantizers designed for variances $\hat{\sigma}_{p}^{2}, p=1, \ldots, k$, that have been log-uniformly distributed in the dynamic range of variances $B=20 \log \left(\sigma_{\max } / \sigma_{\min }\right)$

$$
20 \log \hat{\sigma}_{p}=20 \log \sigma_{\min }+(2 p-1) \frac{B}{2 k},
$$

where $p=1, \ldots, k$.

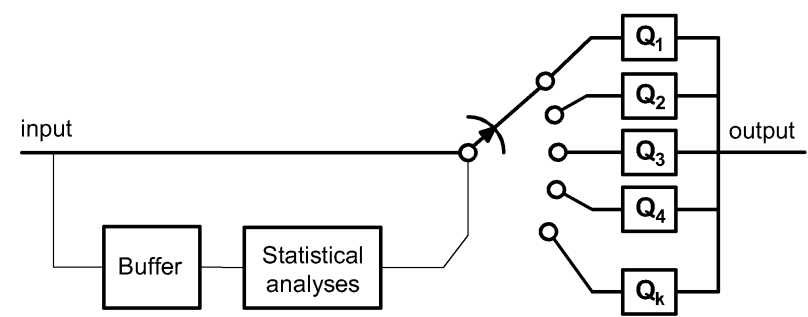

Fig. 2. The switched technique of the adaptive scalar quantization.

With the appliance of switched technique and after the statistical analyses the $s$-th quantizer is chosen among $k$ available ones, whereby a designation where the current frame belongs is unambiguous. The design of each of $k$ available quantizers is performed separately, and it implies the determination of optimal amplitude as

$$
x_{\max }^{p}=c_{\mathrm{opt}} \hat{\sigma}_{p}
$$

with the aim of reaching minimal distortion, i.e. maximal SQNR, in the point $\sigma=\hat{\sigma}_{p}$. For this criterion the optimal parameters of quantizer $c_{\mathrm{opt}}$ and $\mu_{\mathrm{opt}}$ are computed with numerical Nelder-Mead method, as well as maximal SQNR. These values are shown in Table I.

An adaptation technique requires a transmission of 
additional information about the quantizer selected for the processing of current frame sized $M$, out of $k$ available ones, which all have $N$ levels. Bit rate [bits/sample] is equal to

$$
R=\log _{2} N+\frac{\log _{2} k}{M} .
$$

TABLE I. OPTIMAL VALUES FOR DESIGNATED NUMBER OF REPRESENTATION LEVELS $N$.

\begin{tabular}{|c|c|c|c|}
\hline $\boldsymbol{N}$ & $\boldsymbol{c}_{\text {opt }}$ & $\boldsymbol{\mu}_{\text {opt }}$ & SQNR \\
\hline 64 & 3.54 & 3.12 & 31.45 \\
\hline 128 & 3.88 & 3.84 & 37.11 \\
\hline
\end{tabular}

Figure 3 gives SQNR of switched QL quantizer for $k=8$ and $k=16$ quantizers and $N=64$ and $N=128$ levels.

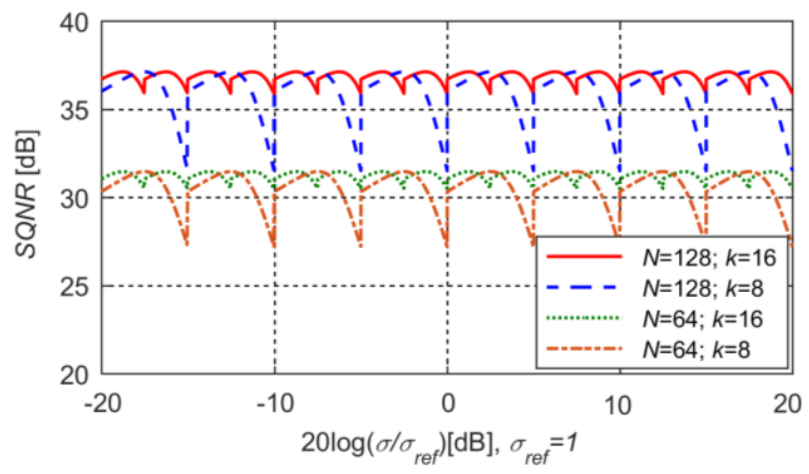

Fig. 3. SQNR of switched QL quantizer.

\section{SWITCHED PIECEWISE UNIFORM QUANTIZER}

Piecewise uniform (PU) quantizer follows above mentioned theoretical settings of QL quantizer. The main difference is in the approximation between samples: QL uses non-linear splines and PU uses linear splines.

In switched technique, as above defined, support region's amplitude is different for every quantizer $p=1, \ldots, k$

$$
x_{\max }^{p}=c_{p} \hat{\sigma}_{p},
$$

where quantizer's relative range factor $c_{p}$ is also different for every quantizer, contrary the parameter in (9) where it is the same for all of $k$ quantizers.

This support region is divided into $2 l=8$ unequal segments whose thresholds are defined as

$$
x_{i}^{p}=\left[(\mu+1)^{i / l}-1\right] \frac{x_{\max }^{p}}{\mu},
$$

where $i=0, \ldots, l$. Inside the segment quantization is uniform with $t=N / 2 l$ cells. The step size of quantization (cell width) is

$$
\Delta_{i}^{p}=\left[(\mu+1)^{(i+1) / l}-(\mu+1)^{i / l}\right] \frac{x_{\max }^{p}}{t \mu},
$$

where $i=0, \ldots, l-1$. The parameter $\mu$ is known in advance. The determination of optimal quantizer's relative range factor $c_{p}$ follows the aforesaid idea linked with (9), i.e. the optimal value should lead to the maximal SQNR. We will use different integer values from $\left[c_{\min }, c_{\max }\right]$ and observe where average value of SQNR reaches maximum. Average SQNR is calculated in the large number of uniformly distributed points $n$ among the wide range of variances $B$, as following

$$
\operatorname{SQNR}_{\mathrm{av}}=\frac{1}{n} \sum_{m=1}^{n} \operatorname{SQNR}\left(\sigma=\sigma_{m}\right) .
$$

In order to compare our results with other proposed solutions, we also calculate $\mathrm{SQNR}_{\max }$ (maximal value) as

$$
\operatorname{SQNR}_{\text {max }}=\max \left(\operatorname{SQNR}\left(\sigma_{1}\right), \ldots, \operatorname{SQNR}\left(\sigma_{n}\right)\right) .
$$

Given formulae for SQNR and distortion still apply. However, granular and overload distortion and calculated in a different way. Granular distortion for piecewise linear quantization and high bit rate is [3]

$$
\begin{aligned}
D_{\mathrm{g}}(\sigma) & =\int_{-x_{\max }^{p}}^{x_{\max }^{p}} \frac{\Delta^{2}(x, \sigma) p(x, \sigma)}{12} d x= \\
& =\frac{1}{12} \sum_{i=0}^{l-1} \Delta_{i}^{2}(\sigma) P_{i}(\sigma),
\end{aligned}
$$

where $\Delta_{i}(\sigma)$ represents cell width, as defined in (13). $P_{i}$ is a cumulative probability of $i$-th segment

$$
P_{i}(\sigma)=\int_{x_{i}^{p}}^{x_{i+1}^{p}} p(x, \sigma) d x=Q\left(x_{i}^{p} / \sigma\right)-Q\left(x_{i+1}^{p} / \sigma\right)
$$

Here used function $Q(x)$ is defined as numerical function

$$
Q(x)=\int_{x}^{\infty} \exp \left(-t^{2} / 2\right) / \sqrt{2 \pi} d t
$$

and we compute it as [7]

$$
Q(x) \cong \exp \left(-x^{2} / 2\right) / \sqrt{2 \pi} \times 4 /\left(3 x+\sqrt{x^{2}+8}\right),
$$

where $x>1$.

$Q(x) \cong \exp \left(-x^{2} / 2\right) / \sqrt{2 \pi} \times \pi /\left((\pi-1) x+\sqrt{x^{2}+2 \pi}\right)$

where $x \leq 1$. On the other side, overload distortion is calculated as [8]:

$$
\begin{aligned}
D_{\mathrm{ov}}(\sigma)=\hat{\sigma}_{p}^{2} \sqrt{\frac{2}{\pi}} \exp \left(-\frac{\left(x_{N}^{p}\right)^{2}}{2 \sigma^{2}}\right) \frac{\rho\left(2 \rho^{4}-2 \rho^{2}+1\right)}{\left(x_{N}^{p} / \hat{\sigma}_{p}\right)^{3}}, \\
x_{N}^{p}=x_{\max }^{p}-\Delta_{l-1}^{p},
\end{aligned}
$$

where $\Delta_{l-1}^{p}$ is defined in (13) and $\rho=\sigma / \hat{\sigma}_{p}$.

The obtained values for $\mathrm{SQNR}_{\mathrm{av}}$ and $\mathrm{SQNR}_{\max }$ of switched piecewise uniform quantizer with $N=128$ levels 
and different values of parameters $k$ and $\mu$ are shown in Table II.

TABLE II. VALUES FOR SQNR ${ }_{a v}[\mathrm{~dB}]$ AND SQNR $\max _{\max }[\mathrm{dB}]$ OF SWITCHED PIECEWISE UNIFORM QUANTIZER WITH $N=128$ LEVELS AND $l=4$ SEGMENTS

\begin{tabular}{|c|c|c|c|}
\hline \multirow{2}{*}{$\boldsymbol{\mu}$} & \multicolumn{2}{|c|}{$\mathbf{S Q N R}_{\mathbf{a v}}[\mathbf{d B}]$} & \multirow{2}{*}{ SQNR $_{\text {max }}[\mathbf{d B}]$} \\
\cline { 2 - 3 } & $\boldsymbol{k}=\mathbf{1 6}$ & $\boldsymbol{k}=\mathbf{8}$ & \\
\hline 3 & 36.673 & 35.931 & 36.921 \\
\hline 7 & 36.436 & 35.764 & 36.602 \\
\hline 15 & 35.541 & 35.315 & 35.683 \\
\hline 31 & 34.194 & 34.214 & 34.305 \\
\hline 63 & 32.855 & 32.808 & 32.889 \\
\hline
\end{tabular}

The bit rate follows the same formula (10). Figure 4 shows the average bit rates for $k=8$ and $k=16$ quantizers and $N=64$ and $N=128$ levels.

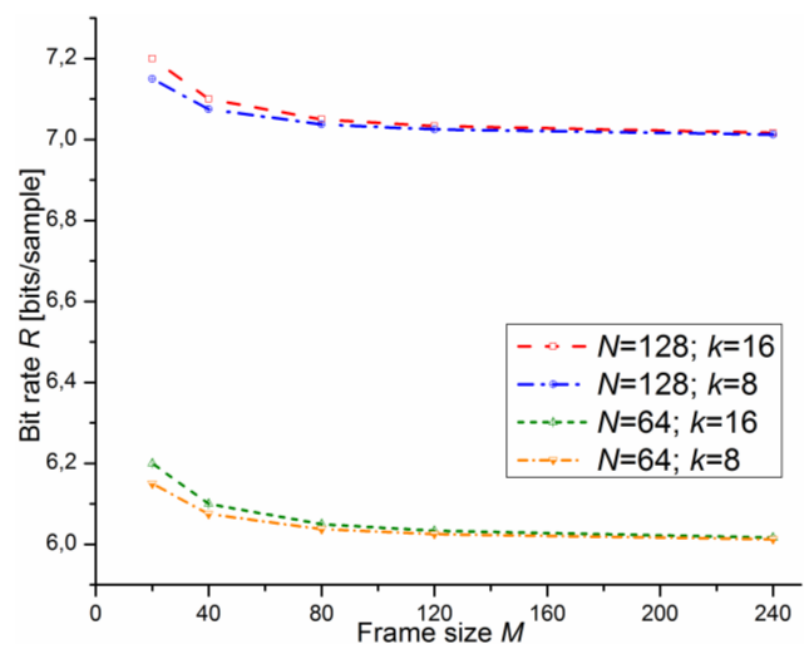

Fig. 4. Bit rate of switched QL quantizer.

\section{EXPERIMENTAL RESULTS}

An analysis of proposed quantizers is based on real speech signals from the ITU-T database [7]. Analysed speech signals are from different speakers talking in English. Signals are labelled as SP01-SP16, whereas first 8 speakers (SP01-SP08) are male and last 8 speakers (SP09-SP16) are female. Signal samples are coded with 16 bits, sampled at frequency equal to $8 \mathrm{kHz}$ and filtered within the range $300 \mathrm{~Hz}-3400 \mathrm{~Hz}$. Switched scalar quasi-logarithmic quantizers (SWLOG) and switched scalar piecewise linear quantizers (SWPPUN) with $2 l=8$ linear segments are analysed.

Optimal values of parameters $c$ and $\mu$, as shown in Table I, are used for the quantizer designing. Simulations are performed for $N=64$ and $N=128$ quantization levels, for switched quantization with $k=8$ and $k=16$ quantizers, and different frame lengths $M$. In Table III are shown values for $N=64, k=16, l=4$. Average values for SQNR, computed in simulations, are shown in Table IV.

\section{CONCLUSIONS}

As expected, larger number of levels $N$, as well as larger number of quantizers $k$, imply higher SQNR. QL quantizer has better performances and higher complexity of hardware realization. For the software realization, recommendation is use QL quantizer, but for hardware realization piecewise uniform quantizer is recommended.
TABLE III. EXPERIMENTALLY OBTAINED VALUES FOR SQNR [dB] OF SWITCHED QUASI-LOGARITHMIC AND PIECEWISE UNIFORM QUANTIZER WITH $N=64$ LEVELS, $k=16$ QUANTIZERS, $l=4$

\begin{tabular}{|c|c|c|c|c|c|c|}
\hline \multirow{2}{*}{ Sample } & \multirow{2}{*}{ Adaptation } & \multicolumn{5}{|c|}{ Frame length $\boldsymbol{M}$} \\
\cline { 2 - 7 } & & $\mathbf{2 0}$ & $\mathbf{4 0}$ & $\mathbf{8 0}$ & $\mathbf{1 6 0}$ & $\mathbf{2 4 0}$ \\
\hline \multirow{2}{*}{ SP01 } & SWLOG & 31.27 & 30.59 & 30.29 & 29.15 & 28.68 \\
\cline { 2 - 7 } & SWPPUN & 31.25 & 30.59 & 30.28 & 29.14 & 28.67 \\
\hline \multirow{2}{*}{ SP02 } & SWLOG & 31.17 & 31.29 & 31.29 & 30.87 & 29.49 \\
\cline { 2 - 7 } & SWPPUN & 31.18 & 31.26 & 31.26 & 30.82 & 29.45 \\
\hline \multirow{2}{*}{ SP03 } & SWLOG & 31.15 & 30.43 & 30.33 & 29.18 & 29.31 \\
\cline { 2 - 7 } & SWPPUN & 31.16 & 30.47 & 30.30 & 29.15 & 29.27 \\
\hline \multirow{2}{*}{ SP04 } & SWLOG & 31.35 & 31.13 & 31.29 & 31.09 & 30.83 \\
\cline { 2 - 7 } & SWPPUN & 31.39 & 31.16 & 31.27 & 31.12 & 30.78 \\
\hline \multirow{2}{*}{ SP05 } & SWLOG & 31.40 & 31.40 & 31.07 & 30.68 & 30.73 \\
\cline { 2 - 7 } & SWPPUN & 31.36 & 31.36 & 31.06 & 30.67 & 30.73 \\
\hline \multirow{2}{*}{ SP06 } & SWLOG & 31.34 & 31.40 & 31.26 & 30.98 & 29.99 \\
\cline { 2 - 7 } & SWPPUN & 31.33 & 31.42 & 31.21 & 30.93 & 29.96 \\
\hline \multirow{2}{*}{ SP07 } & SWLOG & 31.41 & 31.46 & 31.48 & 31.42 & 31.09 \\
\cline { 2 - 7 } & SWPPUN & 31.38 & 31.43 & 31.45 & 31.40 & 31.09 \\
\hline \multirow{2}{*}{ SP08 } & SWLOG & 31.35 & 31.47 & 31.47 & 31.23 & 30.10 \\
\cline { 2 - 7 } & SWPPUN & 31.32 & 31.43 & 31.44 & 31.23 & 30.08 \\
\hline \multirow{2}{*}{ SP09 } & SWLOG & 31.14 & 31.22 & 30.96 & 30.48 & 30.32 \\
\cline { 2 - 7 } & SWPPUN & 31.02 & 31.17 & 30.89 & 30.45 & 30.27 \\
\hline \multirow{2}{*}{ SP10 } & SWLOG & 31.35 & 31.29 & 31.47 & 31.20 & 30.40 \\
\cline { 2 - 7 } & SWPPUN & 31.32 & 31.26 & 31.40 & 31.16 & 30.34 \\
\hline \multirow{2}{*}{ SP11 } & SWLOG & 30.28 & 30.39 & 30.26 & 30.06 & 29.41 \\
\cline { 2 - 7 } & SWPPUN & 30.23 & 30.36 & 30.25 & 30.03 & 29.38 \\
\hline \multirow{2}{*}{ SP12 } & SWLOG & 31.51 & 31.36 & 31.06 & 30.71 & 30.33 \\
\cline { 2 - 7 } SP16 & SWPPUN & 31.49 & 31.33 & 31.04 & 30.72 & 30.33 \\
\hline \multirow{2}{*}{ SP13 } & SWLOG & 31.35 & 31.30 & 31.15 & 30.70 & 30.78 \\
\cline { 2 - 7 } & SWPPUN & 31.26 & 31.24 & 31.10 & 30.64 & 30.74 \\
\hline \multirow{2}{*}{ SP14 } & SWLOG & 31.35 & 31.39 & 31.37 & 31.27 & 30.98 \\
\cline { 2 - 7 } & SWPPUN & 31.30 & 31.40 & 31.36 & 31.25 & 30.98 \\
\cline { 2 - 7 } & SWLOG & 31.34 & 31.33 & 31.30 & 30.93 & 30.24 \\
\cline { 2 - 6 } & SWPPUO & 31.28 & 31.29 & 31.24 & 30.87 & 30.18 \\
\hline \multirow{2}{*}{ SWLOG } & 31.26 & 31.17 & 31.07 & 30.66 & 30.13 \\
\hline & & & & & & \\
& SWPUN & 31.23 & 31.16 & 31.03 & 30.63 & 30.10 \\
\hline
\end{tabular}

TABLE IV. AVERAGE VALUES FOR SQNR [dB].
\begin{tabular}{|c|c|c|c|c|c|c|}
\hline \multirow{2}{*}{ SQNR } & \multirow{2}{*}{ Adaptation } & \multicolumn{5}{c|}{ Frame length $\boldsymbol{M}$} \\
\cline { 3 - 7 } & & $\mathbf{2 0}$ & $\mathbf{4 0}$ & $\mathbf{8 0}$ & $\mathbf{1 6 0}$ & $\mathbf{2 4 0}$ \\
\hline $\begin{array}{c}N=64 \\
k=8\end{array}$ & SWLOG & 30.92 & 30.69 & 30.47 & 29.91 & 29.22 \\
\cline { 2 - 7 } & SWPPUN & 30.90 & 30.67 & 30.45 & 29.90 & 29.22 \\
\hline $\begin{array}{c}N=64 \\
k=16\end{array}$ & SWLOG & 31.26 & 31.17 & 31.07 & 30.66 & 30.13 \\
\cline { 2 - 7 }$N=128$ & SWPPUN & 31.23 & 31.16 & 31.03 & 30.63 & 30.10 \\
\cline { 2 - 7 }$k=8$ & SWLOG & 36.65 & 36.31 & 36.05 & 35.16 & 33.93 \\
\hline \multirow{2}{*}{$\begin{array}{c}N=128 \\
k=16\end{array}$} & SWLOG & 36.56 & 36.21 & 35.95 & 35.07 & 33.86 \\
\cline { 2 - 7 } & SWPPUN & 36.84 & 36.72 & 36.61 & 35.95 & 35.02 \\
\hline
\end{tabular}

A proper comparison for the values of factor $\mu=15,31$, 63 and $k=8,16$ quantizers can be made between this model with $N=128$ levels and a model from [2] with $N=256$ levels. In the latter an experiment with real samples is not performed. Our solution has about $4 \mathrm{~dB}$ higher $\mathrm{SQNR}_{\mathrm{av}}$ in all cases except the $\mu=63$ and $k=8$, in which case it is lower for about $0.2 \mathrm{~dB}$. On the other hand, paper [4] has only experimental results. For smaller number of quantizers (up to $k=16$ ), there is a big deviation between $\mathrm{SQNR}_{\mathrm{av}}$ and $\mathrm{SQNR}_{\max }$, which points to the insufficient reliability of this paper's results. Our solution gives better performances, i.e. approximately $1 \mathrm{~dB}$ higher SQNR, than this paper's best solution, although this model is more complex than ours.

Future researches would direct toward the construction of quantizer and the computation of performances on the mean bit rates (mean quality coding of the speech signal). 


\section{REFERENCES}

[1] Z. Peric, J. Nikolic, "An adaptive waveform coding algorithm and its application in speech coding", Digital Signal Processing, vol. 22, no. 1, pp. 199-209, 2012. DOI: 10.1016/j.dsp.2011.09.001.

[2] N. Vucic, Z. Peric, M. Dincic, "Switched quasi-logarithmic quantizer with golomb-rice coding", Elektronika ir elektrotechnika, vol. 23, no. 4, 2017. DOI: 10.5755/j01.eie.23.4.18727.

[3] N. Vucic, Z. Peric, M. Dincic, "Design of switched quasi-logarithmic quantizer for gaussian source with variable length codes", $13^{\text {th }}$ Int Conf. TELSIKS, pp. 203-206, Nis, Serbia, 2017. DOI: 10.1109/TELSKS.2017.8246263.

[4] L. Velimirovic, S. Maric, "New adaptive compandor for LTE signal compression based on spline approximations", ETRI Journal, vol. 38, no. 3, pp. 463-468, 2016. DOI: 10.4218/etrij.16.0115.0506.

[5] N. S. Jayant, P. Noll, Digital coding of waveforms, principles and applications to speech and video. New Jersey: Prentice Hall, 1984, pp. 115-251.

[6] A. Gasull, F. Utzet, "Approximating Mills ratio", Journal of Mathematical Analysis and Applications, vol. 420, pp. 1832-1853, 2014. DOI: 10.1016/j.jmaa.2014.05.034.

[7] ITU-T Test Signals for Telecommunication Systems, Test Vectors Associated to Rec. ITU-T P.50, 2015. [Online]. Available: http://www.itu.int/ITU-T/dbase.

[8] S. Na, "Asymptotic formulas for variance-mismatched fixed-rate scalar quantization of a gaussian source", IEEE Trans. Signal Processing, vol. 59, no. 5, pp. 2437-2441, 2011. DOI: 10.1109/TSP.2011.2112354 